\title{
C-arm CT: Reconstruction of Dynamic High Contrast Objects Applied to the Coronary Sinus
}

\author{
Christopher Rohkohl, Günter Lauritsch, Alois Nöttling, Marcus Prümmer and Joachim Hornegger
}

\begin{abstract}
For many interventional procedures the 3-D reconstruction of dynamic high contrast objects from $\mathrm{C}$-arm data is desirable. We present a method for compensating artifacts from periodic motions by providing a modified filtered backprojection algorithm. The proposed algorithm comprises three steps: First, the reconstruction of an initial reference volume from a phaseconsistent subset of the projection data. Secondly, the selection of proper data for a motion corrected reconstruction using as many projections as possible in the third step. The first step is addressed by gating in combination with a modified backprojection operator which reduces streak artifacts, the second by analysis of the cardiac motion characteristics and the impact on gated reconstruction quality and the third by accumulating gated sub-reconstructions registered with the reference volume. We present first clinical results from real patient data for the reconstruction of the coronary sinus.
\end{abstract}

\section{INTRODUCTION}

$\mathbf{T}$ HE 3-D reconstruction of cardiac vascular systems like the coronary sinus, coronary arteries or other dynamic high contrast structures using C-arm CT is highly desirable for supporting $\mathrm{C}$-arm based interventions like pacemaker implantations or ablation procedures. This paper considers the reconstruction of the venous cardiac vascular system, especially the coronary sinus and its tributaries.

\section{A. Clinical Background}

The knowledge of the anatomy of the coronary sinus and its tributaries is important during the implantation of biventricular pacemakers. Those are commonly utilized if the synchronization of the electrical activity of the left and right heart is defective. By placing an additional pacing lead via the coronary sinus into a left ventricular cardiac vein, the electrical signals can be resynchronized by simultaneous stimulation of the right and left ventricle [1], [2]. The localization of a proper lead location in a left ventricular cardiac vein is very important for the success of the resynchronization therapy. The usage of additional 3-D information can help finding good locations for lead placement and can help saving contrast agent using 2-D/3-D overlay techniques during the intervention.

\section{B. Technical Problems and State-of-the-Art}

The reconstruction methods for 3-D volumes from 2-D projections rely on the assumption of motionless subjects.

C. Rohkohl, M. Prümmer and J. Hornegger are with the Chair of Pattern Recognition, FA University Erlangen-Nuremberg, Germany (c.rohkohl@oneder.de, \{pruemmer, hornegger\}@informatik.uni-erlangen.de).

C. Rohkohl, G. Lauritsch and A. Nöttling are with the Siemens AG, Healthcare Sector, Forchheim, Germany (\{guenter.lauritsch, alois.noettling\}@siemens.com).
This obviously does not hold for dynamic objects as the heart. In cardiac applications a steady periodic motion of the heart complicates a 3-D reconstruction. A common way to approach the problem of cardiac motion for C-arm short-scan reconstructions is a gating of the projection data according to the electrocardiogram, i.e. only a subset of the data acquired in the same object motion state is used for reconstruction using analytical [3], [4], [5] or iterative methods [6], [7].

Another possibility is the compensation of the object motion during reconstruction. For that an estimate of the object motion is required which is commonly obtained by registering symbolic reconstructions of different motion phases [8], [9], [10].

Both approaches have limitations: for single sweep acquisitions gating leads to missing data which causes streak artifacts. Motion estimation from fully automatic symbolic reconstructions requires a good segmentation of the projection images which is yet a challenging problem in a clinical environment.

\section{Paper Overview}

In this paper, a novel approach for gated reconstruction, motion estimation and compensation is presented. A voxeldependent weighting scheme of a Feldkamp-like reconstruction is proposed which reduces streak artifacts and significantly increase the reconstruction quality. The optimized gated and streak reduced reconstruction is then used in a fast and highly parallelizable combined motion estimation and compensation algorithm which is data-driven and does not require preliminary symbolic reconstructions.

\section{Methods}

\section{A. Overview}

The proposed method for reconstructing dynamic high contrast objects comprises three steps:

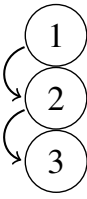

\section{Gated reference reconstruction}

Phase-consistent selection of projection data

Combined motion estimation and compensation

Each projection image $p_{\gamma}$ acquired at the acquisition angle $\gamma \in\left[0, \gamma_{\max }\right]$ is assigned a motion phase $\phi(\gamma)$. The motion phase $\phi(\gamma) \in[0,1]$ of a projection image $p_{\gamma}$ denotes the relative heart phase according to the R-peaks of the electrocardiogram. A reconstruction of a specific motion phase is done using a gating function $\lambda:\left[0, \gamma_{\max }\right] \mapsto[0,1]$. A gating 
function selects a subset of the projection data by assigning a weighting value. All zero-weighted projection images are ignored and non-zero weighted projections are used for a sparse reconstruction at a specific motion phase. The quality of a gated reconstruction depends on two factors. First, the amount of missing data and second, the consistency of the projection data used for reconstruction. In this paper, the term consistency of projection data is used to describe the magnitude of object motion present in the projection data (or a subset of the projection data). Projection data with small object motion, i.e. data from similar motion phases, is more consistent than data containing strong object motion, i.e. data from different motion phases.

In subsection $C$, the first algorithm step, i.e. the creation of a reference reconstruction at a specific motion phase including a novel streak artifact reduction technique is presented.

A gated reconstruction can be performed for several different motion phases. As only a subset of the projection data is used for a gated reconstruction, the goal is to use more data from different motion phases for a reconstruction at a specific reference motion phase. This is done by estimating the motion between the reference phase and other motion phases. The selection of the data, i.e. the gating functions, used for the motion corrected reconstruction is of high importance. How to select this data is part of algorithm step 2 which is presented in subsection $D$.

The estimated motion is then used to correct the data used for the reconstruction in order to perform a motion compensated reconstruction with more projection data than in the reference reconstruction of the first algorithm step. A fast and highly parallelizable method for motion estimation and compensation is part of algorithm step 3 and details can be found in subsection $E$.

\section{B. Gated Short-Scan FDK Reconstruction}

1) Reconstruction Algorithm: The Feldkamp algorithm (FDK) [11] is a wide-spread analytic reconstruction method for cone beam reconstruction on circular trajectories. It is a backprojection based algorithm where the ramp-filtered and weighted projection data is backprojected for reconstruction. In a gated reconstruction the data is additionally weighted using a gating function $\lambda$.

For a voxel $\boldsymbol{x}$ and a gating function $\lambda$ this leads to the following reconstruction formula for a gated short-scan (GSS) reconstruction:

$$
f_{\mathrm{GSS}(\lambda)}(\boldsymbol{x})=\int_{0}^{\gamma_{\max }} \lambda(\gamma) \cdot \Upsilon(\boldsymbol{x}, \gamma) d \gamma,
$$

with $\Upsilon(\boldsymbol{x}, \gamma)$ being the post-weighted and back-projected value of the filtered projection image acquired at angle $\gamma \in$ $\left[0, \gamma_{\max }\right]$. The individual voxel contribution $\Upsilon(\boldsymbol{x}, \gamma)$ is given by

$$
\Upsilon(\boldsymbol{x}, \gamma)=\frac{R_{F}^{2}}{\left(R_{F}+\boldsymbol{x} \cdot \hat{\boldsymbol{s}}\right)^{2}} \cdot p_{\gamma}^{F}(u(\boldsymbol{x}, \gamma), v(\boldsymbol{x}, \gamma)) .
$$

The notation is adapted from [12], with $R_{F D}$ being the distance between the source focus and the detector, $R_{F}$ being

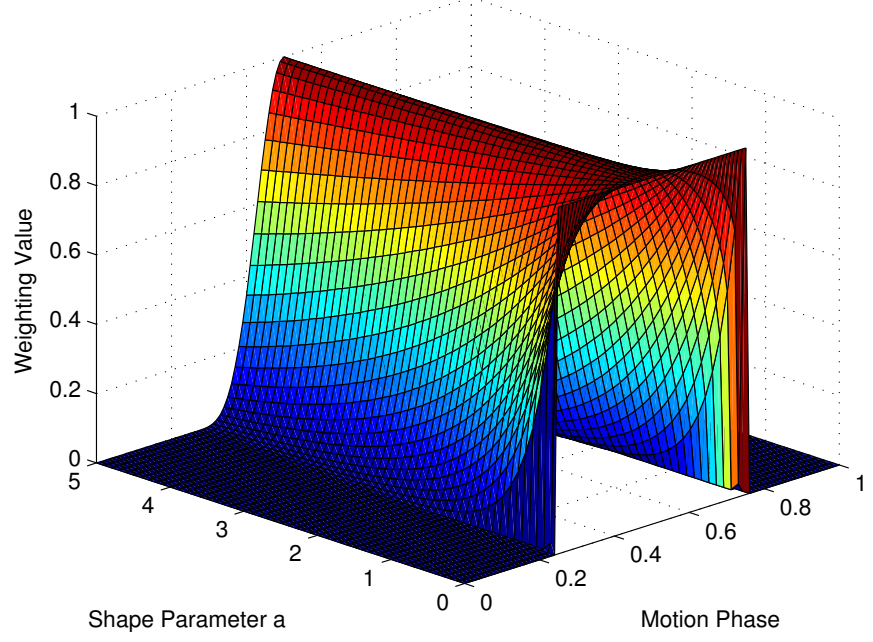

Fig. 1: Generalized weighting function $\lambda_{\phi_{\text {ref }}}$ for various shape parameters $a$, given a reference motion phase of $\phi_{\text {ref }}=0.5$ and a window width of $w=0.5$.

the the distance from the source focus to the rotational center and $\hat{s}$ being the unit vector pointing from the source focus to the center of the detector. The image coordinates are denoted $(u, v)$ with the horizontal component being parallel to the circular plane. The filtered projection data is given by

$p_{\gamma}^{F}(u, v)=\left(\frac{R_{F D}}{\sqrt{\left(R_{F D}^{2}+u^{2}+v^{2}\right)}} W_{\mathbf{r}}(\gamma, u, v) p_{\gamma}(u, v)\right) * h(u)$

with $h$ being the ramp-filter and $W_{\mathrm{r}}$ being the redundancy weighting for identical rays.

2) Gating Function: For the selection of a gating function $\lambda$, Schäfer et al. [3] proposed the usage of a smooth weighting function for the reconstruction of the coronary arteries which is based on a squared cosine function. In this work a more general family of cosine-based gating functions is proposed. For a window width $w$, a shape parameter $a$ and a reference motion phase $\phi_{\text {ref }}$ the gating function is given by

$$
\lambda_{\phi_{\mathrm{ref}}}^{a, w}(\gamma)= \begin{cases}\cos ^{a}\left(\frac{\delta\left(\phi(\gamma), \phi_{\mathrm{ref}}\right)}{w} \pi\right) & \text { if } \delta\left(\phi(\gamma), \phi_{\mathrm{ref}}\right) \leq \frac{w}{2} \\ 0 & \text { otherwise } .\end{cases}
$$

The distance function which measures the distance between two motion phases is denoted $\delta$. For a relative heart phase it is defined as $\delta\left(\phi_{1}, \phi_{2}\right)=\min _{c \in\{0,1,-1\}}\left|\phi_{1}-\phi_{2}+c\right|$. The non-zero support region of the weighting function is characterized by the window width $w \in(0,1]$. The shape of the support region around the central reference motion phase $\phi_{\text {ref }}$ is determined by a shape parameter $a \geq 0$. In figure 1 the gating function is depicted for various shape parameters at a fixed window width $w=0.5$. For $a=0$ the gating window is a rectangle while with increasing $a$ the impact of projections close to the reference phase gets larger. The gating function proposed by Schäfer et al. [3] is obtained using the shape parameter $a=2$. 


\section{Step 1: Gated Reference Reconstruction}

The goal of the first algorithm step is to obtain a reference reconstruction at a specific motion phase which later on in the third step (section $E$ ) is used for motion estimation. The quality of this reference reconstruction is crucial for a successful motion estimation. It is obtained using a version of the gated FDK reconstruction presented in the previous section, modified by a new weighting term for reducing streak artifacts.

1) Streak-Reduced Gated Short-Scan Reconstruction: For a gated reconstruction only a small number of projection images is available. After ramp-filtering of the projection images each voxel receives view-dependent contributions that are summed up in order to reconstruct the intensity value. In the presence of high contrast objects very high and low contributions are observed for voxels of actually low intensity. These high and low view-dependent contributions cannot be canceled out by other projection views as it is normally the case due to the missing data. This fact is manifested in terms of streak artifacts (very low and high intensity streaks) in the reconstructed volume.

The general idea is to weight the view-dependent voxel contributions based on their contribution value for each voxel separately. The aim is to design a weighting scheme in such a way that the highest and lowest contributions are weighted down. Weighting-down of highest contributions reduces streak artifacts at voxels of low intensity whereas weighting-down of the lowest contributions dimishes signal reduction at voxels of high intensity.

Formally, the standard reconstruction formula (1) is modified as follows to obtain the gated streak reduced short-scan (GSRSS) reconstruction:

$$
f_{\mathrm{GSRSS}(\lambda, W)}(\boldsymbol{x})=\nu \int_{0}^{\gamma_{\max }} W(q(\boldsymbol{x}, \gamma)) \cdot \lambda(\gamma) \Upsilon(\boldsymbol{x}, \gamma) d \gamma
$$

The function $q\left(\boldsymbol{x}, \gamma_{0}\right)$ returns the proportion of contributions for the voxel $\boldsymbol{x}$ that are smaller than the single contribution from angle $\gamma_{0}$. It is given by

$$
q\left(\boldsymbol{x}, \gamma_{0}\right)=\frac{1}{\gamma_{\max }} \int_{0}^{\gamma_{\max }} H\left(\lambda\left(\gamma_{0}\right) \Upsilon\left(\boldsymbol{x}, \gamma_{0}\right)-\lambda(\gamma) \Upsilon(\boldsymbol{x}, \gamma)\right) d \gamma
$$

with $H(\cdot)$ being the Heaviside step function [13].

The weighting function $W:[0,1] \mapsto[0,1]$ can be selected arbitrary. For streak suppression, as previously explained, the smallest and largest contributions should receive a lower weighting than medium values. In this paper, the same family of cosine functions already presented in the previous section will be used. It is given by

$$
W_{a}^{w}(x)= \begin{cases}\cos ^{a}\left(\frac{|0.5-x|}{w} \pi\right) & \text { if }|0.5-x| \leq \frac{w}{2} \\ 0 & \text { otherwise }\end{cases}
$$

and parameterized by a shape parameter $a \geq 0$ and a support window width $w \in(0,1]$. For $w=1$ and $a=0$ (5) simplifies to the standard gated Feldkamp formula (1).
The normalization factor $\nu$ in front of the integral is given by

$$
\nu=\frac{\gamma_{\max }}{\int_{0}^{\gamma_{\max }} W(q(\boldsymbol{x}, \gamma)) d \gamma}
$$

The presented weighting scheme is performed for every voxel independently. Thus every voxel value may be theoretically reconstructed from differing projection images. As an example in figure 2 a standard gated reconstruction of a synthetic phantom is compared to a streak reduced reconstruction.

2) Preparation For Motion Estimation: The gated and streak reduced reference reconstruction is used as input to the motion estimation and compensation in algorithm step three (section $E$ ). The proposed motion estimation scheme is based on a 3-D/3-D registration requiring the high contrast object in the reference reconstruction to be segmented with the intensity of a voxel indicating the likelihood of belonging to the desired object. This segmentation can be done by applying a transfer function automatically or in volume rendering by user interaction. The post-processed reference reconstruction will in the following be denoted $f_{\text {ref }}$.

\section{Step 2: Phase-Consistent Selection of Projection Data}

The main goal of motion estimation and correction (step three) is to utilize more projection data in order to increase the reconstruction quality in comparison to the gated reference reconstruction created in step one. The question that has to be addressed in algorithm step two is how to select this additional data in a meaningful way in a realistic situation.

The problem of selecting subsets of the projection data for the motion estimation and correction is formalized by introducing a set of gating functions $\Lambda=\left\{\lambda_{1}, \ldots, \lambda_{k}\right\}$. Each gating function $\lambda \in \Lambda$ describes a subset of the projection data that is to be used for motion estimation and correction in algorithm step three.

The quality of a gated reconstruction largely depends on the consistency of the projection data. Each of the gating functions should select data that is as consistent as possible. The degree of consistency depends largely on the object motion velocity. In phases of rest a gating is more consistent than in phases of rapid motion.

In a real-world application, e.g. in cardiac imaging, the true object motion is unknown. However, from physiology [14] or measurements using a CT scanner [15] general properties of the object motion can be derived and phases of rest or rapid motion can be identified. In cardiac imaging the motion characteristics are heart rate dependent. For low heart rates $(<85 \mathrm{bpm})$ two phases of rest can be identified. A shorter period of rest in the systole $(25-30 \%$ relative heart phase) and a longer one in the diastole $(70-85 \%$ relative heart phase). With increasing heart rate the diastolic phase shortens drastically while the length of the systolic phase stays approximately constant. In-between those phases the heart performs rapid movements with high velocities.

For composing the set of gating functions this means that periods of rest can be included using large gating windows 


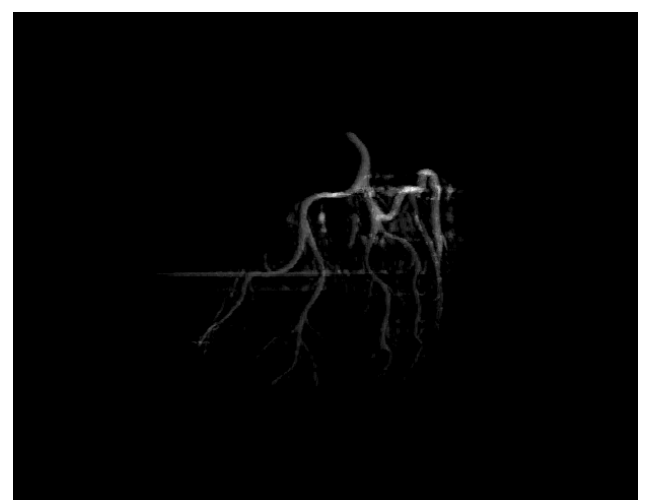

(a) Standard reconstruction

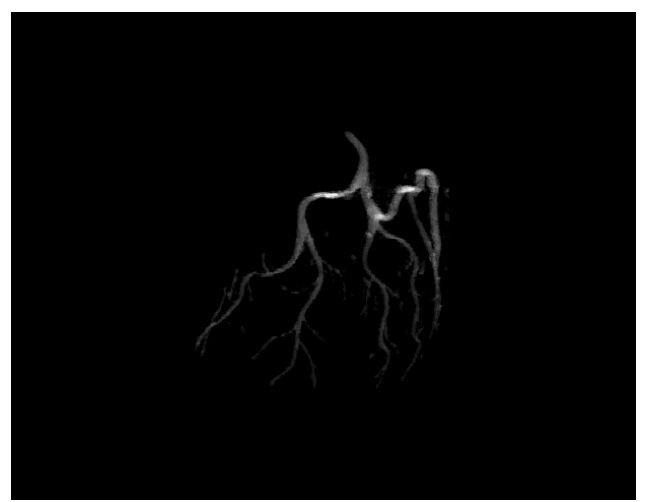

(b) Streak reduced reconstruction

Fig. 2: Illustration of the quality gain obtained by utilizing the streak reduced reconstruction. The example reconstruction results are for a synthetic cardiac vasculature phantom. The gating function parameters were set to $w=0.1, a=2$. The streak reduction was carried out using the weighting function parameters $w=0.7$ and $a=0$.

and periods of rapid motion should be included using a small gating window or be excluded at all.

In figure 3 two possible sets of gating functions for cardiac applications are depicted. The black line shows the mean velocity of points located on the coronary tree dependent on the motion phase according to [15]. As can be seen the velocity curve has two troughs, i.e. phases of rest. The rectangular gating functions depicted in (a) are equidistantly spaced and cover all available projection images. Thus no special care about the reliability of the projection data belonging to each gating window has been taken. This can be sufficient as long as there are enough projection images and the gating window width is small enough. The set of gating functions in (b) respects the velocity information and has larger window widths at the troughs than in phases of rapid motion.

\section{E. Step 3: Combined Motion Estimation and Compensation}

The basic process of performing a motion corrected tomographic reconstruction consists of three steps. First, it has to be decided for a motion model. Based on the parameterization of this model the motion needs to be estimated for each motion phase which is used for a motion compensated reconstruction.

1) Motion Model: An object motion from a state $\phi_{1}$ to another state $\phi_{2}\left(\phi_{1} \mapsto \phi_{2}\right)$ can be characterized by assigning to each voxel in the first motion phase $\boldsymbol{x}^{\phi_{1}}$ a displacement vector. The complete transformation depends on some set of parameters $\Theta$.

A motion model $\mathcal{M}_{\phi_{1}}^{\phi_{2}}: T \times \mathbb{R}^{n} \mapsto \mathbb{R}^{n}$ is a parametric mapping which maps a point $\boldsymbol{x} \in \mathbb{R}^{n}$ to a displacement vector based on the set of parameters $\Theta \in T$. The displacement vector describes the positional change of the point $\boldsymbol{x}$ from motion state $\phi_{1}$ to the motion state $\phi_{2}$ parameterized by $\Theta$, i.e. such that the following holds:

$$
\boldsymbol{x}^{\phi_{2}}=\boldsymbol{x}^{\phi_{1}}+\mathcal{M}_{\phi_{1}}^{\phi_{2}}\left(\Theta, \boldsymbol{x}^{\phi_{1}}\right) \text {. }
$$

A plurality of different motion models can be found in literature, varying from 2-D up to 4-D parameterizations with different degrees of freedom. Cardiac motion is inherently characterized by a non-uniform deformation. In this paper a
3-D B-spline motion model is utilized which is popular in cardiac reconstruction literature. It is given by

$$
\mathcal{M}_{\phi_{1}}^{\phi_{2}}\left(\left\{\boldsymbol{d}_{i j k}\right\}, \boldsymbol{x}\right)=\sum_{i, j, k} B_{i, p}\left(x_{0}\right) B_{j, p}\left(x_{1}\right) B_{k, p}\left(x_{2}\right) \boldsymbol{d}_{i j k} .
$$

The set of uniformly distributed control points is indexed by $i, j, k$ and the corresponding displacement values are given by $\Theta=\left\{\boldsymbol{d}_{i j k}\right\}$. The set of $p$-th degree B-spline basis functions is denoted $\{B \cdot, p\}$ [16].

Cubic B-spline functions are widespread for cardiac motion parameterization in 2-D [17], 3-D [8] and 4-D [9]. If the number of control points equals the number of image voxels, each voxel is assigned an individual displacement vector. Such a model was used in cardiac imaging e.g. by Prümmer et al. [18].

2) Motion Estimation: The estimation of the model parameters is formulated in terms of a 3-D/3-D registration problem where the sparse reference reconstruction $f_{\text {ref }}$ and the gated reconstruction $f_{\mathrm{GSS}(\lambda)}$ need to be aligned. Thus, a set of parameters $\Theta$ minimizing an energy functional $F$ is sought. Formally this problem is stated as

$$
\hat{\Theta}=\arg \min _{\Theta} F\left(f_{\mathrm{ref}}, f_{\mathrm{GSS}(\lambda)}, \Theta\right) .
$$

In this paper, a new energy function consisting of three additive terms is proposed:

$$
\begin{aligned}
F\left(f_{\mathrm{ref}}, f_{\mathrm{GSS}(\lambda)}, \Theta\right) & =\alpha_{J} J\left(f_{\mathrm{ref}}, f_{\mathrm{GSS}(\lambda)}, \Theta\right) \\
& +\alpha_{B} B\left(f_{\mathrm{ref}}, f_{\mathrm{GSS}(\lambda)}, \Theta\right) \\
& +\alpha_{R} R(\Theta) .
\end{aligned}
$$

It consists of two terms $J$ and $B$ measuring the quality of the alignment and a regularization term $R$. The first term $J$ measures how much intensity is concentrated inside the

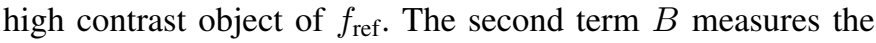
intensity that is concentrated in the border area around the high

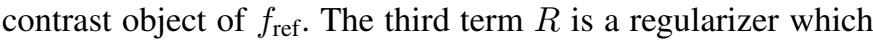
depends on the motion model parameters only. The relative impact of the metric components is determined by the scalars $\alpha_{J} \leq 0, \alpha_{B} \geq 0$ and $\alpha_{R}$. If the two volumes are perfectly aligned the energy functional $F$ will be minimized. 


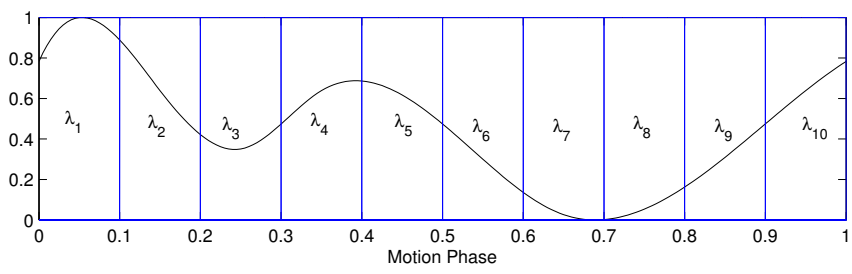

(a)

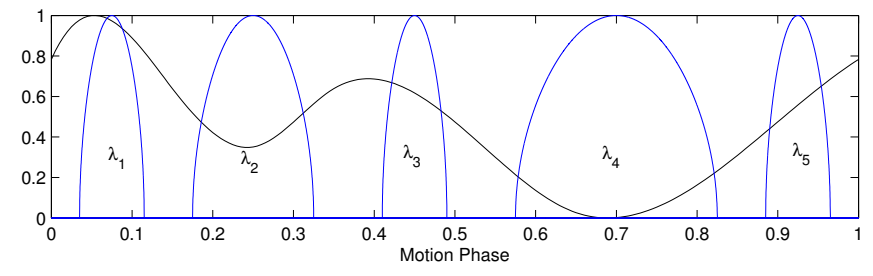

(b)

Fig. 3: Illustration of possible gating functions for cardiac imaging. The black line shows the relative mean velocity of points located on the coronary artery tree depending on the relative heart phase according to [15]. (a) shows a set of 10 equidistantly spaced rectangular gating functions including all projection images. (b) shows a set of 5 different gating functions whose characteristics vary depending on the velocity of the concerning motion phase.

Assuming $\boldsymbol{x}^{\prime}=\boldsymbol{x}+\mathcal{M}_{\phi_{\mathrm{ref}}}^{\phi_{\lambda}}(\Theta, \boldsymbol{x})$, the components of $F$ are formally given by:

$$
\begin{aligned}
& J\left(f_{\mathrm{ref}}, f_{\mathrm{GSS}(\lambda)}, \Theta\right)=\nu_{J} \sum_{\left\{\boldsymbol{x} \mid f_{\mathrm{ref}}(\boldsymbol{x})>0\right\}} f_{\mathrm{ref}}(\boldsymbol{x}) \cdot f_{\mathrm{GSS}(\lambda)}\left(\boldsymbol{x}^{\prime}\right), \\
& B\left(f_{\mathrm{ref}}, f_{\mathrm{GSS}(\lambda)}, \Theta\right)=\nu_{B} \sum_{\left\{\boldsymbol{x} \mid f_{\mathrm{ref}}^{\mathrm{b}}(\boldsymbol{x})>0\right\}} f_{\mathrm{ref}}^{\mathrm{b}}(\boldsymbol{x}) \cdot f_{\mathrm{GSS}(\lambda)}\left(\boldsymbol{x}^{\prime}\right)
\end{aligned}
$$

with the normalizing factors $\nu_{J}=\frac{1}{\left|\left\{\boldsymbol{x} \mid f_{\text {ref }}(\boldsymbol{x})>0\right\}\right|}$ and $\nu_{B}=$ $\frac{1}{\left|\left\{\boldsymbol{x} \mid f_{\text {ref }}^{\mathrm{b}}(\boldsymbol{x})>0\right\}\right|}$.

The volume $f_{\text {ref }}^{\mathrm{b}}$ is a boundary map which has non-zero values at the boundary of non-zero voxels of $f_{\text {ref. }}$. The creation of a boundary map for the reference volume is done by convolving $f_{\text {ref }}$ with a Gauß kernel of standard deviation $\sigma$. All voxels that have a zero value in the original and a non-zero value in the smoothed volume are considered as a boundary voxel. The value of a boundary voxel is the maximum value of $f_{\text {ref }}$ minus the convolved voxel value. All non-border voxels have a value of zero. An example for this process for a 2-D image is presented in figure 4.

The terms $J$ and $B$ of the energy function can be derived analytically and thus can be used for advanced derivative based optimization procedures.

During the estimation of the motion model it needs to be ensured that only plausible deformations are considered, i.e. the deformation field should be smooth and the degree of object motion should be as small as possible. For the B-spline motion model there are two options for regularization. If the number of control points is kept small only smooth deformations are possible. However, for obtaining a larger flexibility of the motion model more control points are required and a regularization term $R$ needs to be supplied.

The following additional regularization term $R$ of the energy functional $F$ is proposed:

$$
\begin{aligned}
R\left(\left\{\boldsymbol{d}_{i j k}\right\}\right) & =\alpha_{1} \sum_{i, j, k}\left(\boldsymbol{d}_{i j k}\right)^{2} \\
& +\alpha_{2} \sum_{i, j, k} \sum_{\substack{\left(i^{\prime}, j^{\prime}, k^{\prime}\right) \\
\in \mathcal{N}_{6}(i, j, k)}}\left(\boldsymbol{d}_{i j k}-\boldsymbol{d}_{i^{\prime} j^{\prime} k^{\prime}}\right)^{2} .
\end{aligned}
$$

This regularization consists of two terms. The first one computes the sum of the lengths of the deformation vectors of the control points. Thus it enforces small motions. The second sums up for each control point the difference to its six closest neighboring control points $\mathcal{N}_{6}$. Hence it enforces a smooth deformation field.

For optimization of the motion model parameters $\left\{\boldsymbol{d}_{i j k}\right\}$ the ITK [19] implementation of the L-BFGS-B algorithm [20] has been used. It requires the first derivative of the energy functional which can be derived analytically.

3) Motion Compensation: In this paper the approach proposed by Schäfer et al. [3] and Prümmer et al. [18] for performing a motion compensated FDK reconstruction is used. Formally the motion compensated gated short-scan FDK (MCGSS) is given by:

$$
f_{\operatorname{MCGSS}(\lambda, \Theta)}(\boldsymbol{x})=\int_{0}^{\gamma_{\max }} \lambda(\gamma) \cdot \Upsilon\left(\boldsymbol{x}+\mathcal{M}_{\phi(\gamma)}^{\phi_{\mathrm{ref}}}(\Theta, \boldsymbol{x}), \gamma\right) d \gamma
$$

Incorporating the presented motion estimation scheme and the selected subsets of projection data into (15) we formally get the following motion estimation and motion compensating (MEMC) reconstruction formula for a given set of gating functions $\Lambda$ :

$$
f_{\operatorname{MEMC}(\Lambda)}(\boldsymbol{x})=\sum_{\lambda \in \Lambda} f_{\operatorname{MCGSS}\left(\lambda, \hat{\Theta}_{\lambda}\right)}(\boldsymbol{x})
$$

with

$$
\hat{\Theta}_{\lambda}=\arg \min _{\Theta} F\left(f_{\text {ref }}, f_{\mathrm{GSS}(\lambda)}, \Theta\right)
$$

The proposed reconstruction formula $f_{\text {MEMC }}$ (16) assumes a gating function dependent motion model, i.e. for all motion phases that are included by a gating window function the same motion model is assumed. This corresponds to a nearest neighbor interpolation scheme for motion phases not being the reference phase of the gating function. Of course other interpolation schemes may be used, e.g. linear interpolation. However, the dominating factor for the reconstruction quality currently is not the interpolation. A proper reference reconstruction and the selection of consistent subsets of projection data which enable a good motion estimation, are the limiting factors. Thus the study of different interpolation schemes is not considered any further in this paper. 


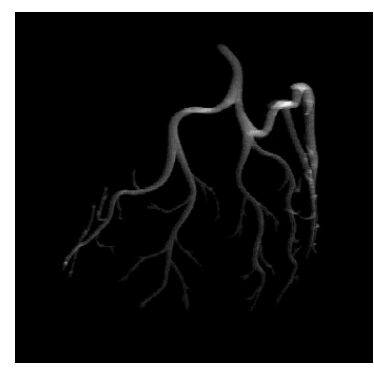

(a) $f_{\text {ref }}$

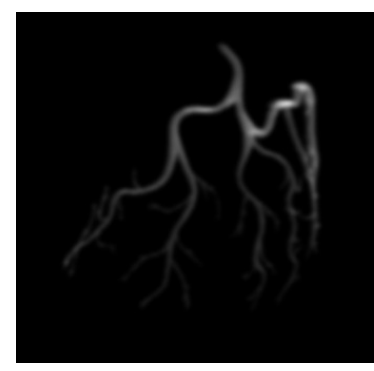

(b) Smoothed $f_{\text {ref }}$ with $\sigma=2$

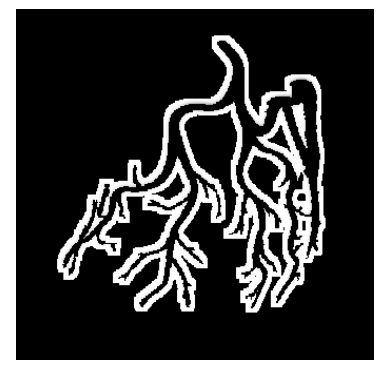

(c) $f_{\text {ref }}^{\mathrm{b}}$

Fig. 4: Example for a boundary map $f_{\text {ref }}^{\text {b }}$ created for a reference volume $f_{\text {ref }}$ which consists of a high contrasted vessel tree.

4) Performance Considerations: The calculation of a metric value is very fast as only non-zero voxels of the sparse reference reconstruction and the corresponding sparse boundary volume are considered, i.e. the sets $\left\{\boldsymbol{x} \mid f_{\text {ref }}(\boldsymbol{x})>0\right\}$ and $\left\{\boldsymbol{x} \mid f_{\text {ref }}^{\mathrm{b}}(\boldsymbol{x})>0\right\}$ do only contain few elements. For the reconstruction of sparse high contrast objects as cardiac vessels more than $99 \%$ of the voxels can be ignored during the evaluation of a metric value and its derivatives.

The code implementation of $f_{\text {MEMC }}$ is straight forward and only requires a small adjustment of the standard reconstruction. Each gating function $\lambda \in \Lambda$ has a set of non-zero $(\lambda>0)$ contributing projection images during a gated reconstruction. For each $\lambda$ those projection images are processed using standard filtering and back-projection. The resulting reconstruction is then used to estimate the motion model parameters. The gated reconstruction is then warped via resampling according to the motion model. This is done for every $\lambda$ and the resampled reconstructions are summed up to get the final reconstruction. This implementation scheme is very fast as only the registration and resampling is added for every gating function. It is also highly parallelizable as the reconstruction and registration can be done for every $\lambda$ independently. The interpolation of image values is done by cubic B-spline interpolation [16].

\section{Clinical Results}

The method was applied to a couple of clinical datasets showing the contrasted coronary sinus. Image acquisition was performed using an Artis zee system (Siemens AG, Healthcare Sector, Forchheim, Germany). The acquisition times were $4 \mathrm{~s}$ to 8 s capturing 191 to 397 projection images. The gating function parameters were set to $a=1$ and $w=0.1$ for the reference reconstruction. The weighting function parameters for streak reduction were set to $a=0$ and $w=0.9$. The set of gating functions for motion estimation and compensation was selected motion specific as presented in figure $3 \mathrm{~b}$ with the gating parameters $a=1$.

The control point spacing of the B-splines was set to $2 \mathrm{~cm}$. The reconstructed volumes were reconstructed with an isotropic voxel size of $1 \mathrm{~mm}$. The parameters for the computation of the energy functional $F$ were set to $\alpha_{1}=\alpha_{2}=0.01$, $\alpha_{J}=-1, \alpha_{B}=1.2, \alpha_{R}=1$ and $\sigma=0.75$.

Three examples are discussed in the following subsections. The first and second one differ by the heart rate. In both cases the heart cycle time is quite constant with low heart rate variability. Due to the constant heart rate the relative ECG phase corresponds to the same physiological heart phase in all cycles. In case of varying cycle times there might be a mismatch since the changes of the physiological phase durations do not scale linearly. To cover such a demanding scenario the third case shows a high heart rate variability. The image quality is evaluated visually regarding contrast homogenity, surface smoothness, and visualization of finer vascular structures.

The reconstruction results for all cases are depicted in figure 6.

\section{A. Heart Rate: $\varnothing 65$ bpm, $\sigma=1.3$ bpm, Best Phase: 75\%}

In the gated reconstruction some parts of the vascular tree are missing in volume rendering due to the incomplete data. Even the large vessel is partly disconnected. This cannot be directly seen from the forward projection depicted in figure 6 . A part of the vasculature indicated by the yellow arrow has been zoomed in and is displayed in figure 5 for comparison. It can be clearly seen that the motion compensated reconstruction is less noisy and fully connected. In addition more small vessels are visible compared to the gated reconstruction.

\section{B. Heart Rate: $\varnothing 91$ bpm, $\sigma=1.1$ bpm, Best Phase: 77\%}

The diastolic phase of rest shortens with the increase of heart rates. In C-arm CT this is not problematic since good temporal resolution is achieved by using information of each heart beat. The motion compensated reconstruction (figure 6) again provides superior quality to the gated reconstruction in terms of noisiness and completeness of the vascular tree in volume rendering.

\section{Heart Rate: $\varnothing 65$ bpm, $\sigma=12.0$ bpm, Best Phase: 35\%}

The higher heart rate variability of this case causes a reduced temporal resolution of the gated reconstructions. Consequently the motion estimation accuracy is lowered. In the last column of figure 6 this can be seen at the main vessel which is more blurred than in the gated reconstruction. However, the gated reconstruction is highly disconnected at some parts of the vascular tree and more details can be observed in the motion compensated reconstruction. 


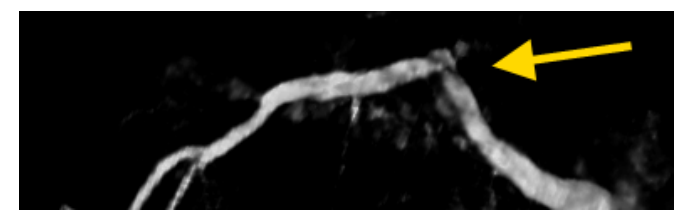

(a) Gated reconstruction

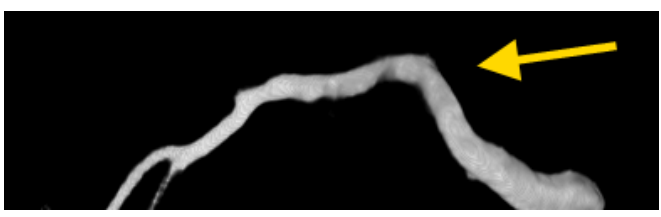

(b) Motion compensated reconstruction

Fig. 5: Comparison of a zoomed in view in volume rendering of the first case presented in the first column of figure 6 . The yellow arrow indicates the location of the extracted position in the forward projection of figure 6 . This is a differing view from which artifacts of the reconstruction can be better observed.

\section{CONCLUSiON}

The presented approach improves the reconstruction quality of dynamic high contrast objects like the coronary sinus significantly in terms of surface smoothness and visualization of finer vascular structures using a single-sweep C-arm acquisition. For three clinical cases the method was demonstrated to handle a wide range of patient heart rates and can even deal with variable cycle times. For the reconstruction only fast FBP-based methods are used. Special care has been taken for the reference reconstruction in order to optimize the motion estimation quality. This was achieved by introducing a new streak reduction technique and a segmentation of the gated reference reconstruction. Further, a motion specific selection of sub-reconstructions improves consistency and accuracy for the fast 3-D/3-D registration using a narrow banding approach which is purely data driven, fast and highly parallelizable.

\section{ACKNOWLEDGMENT}

We thank Prof. Dr. med. J. Brachmann, Dr. med. G. Nölker, Dr. med. H. Marschang and Dr. med. K. Gutleben from the Klinikum Coburg, Germany for providing clinical image data.

\section{DISCLAIMER}

The concepts and information presented in this paper are based on research and are not commercially available.

\section{REFERENCES}

[1] C. Leclercq and D. A. Kass, "Retiming the failing heart: principles and current clinical status of cardiac resynchronization," J Am Coll Cardiol, vol. 39, no. 2, pp. 194-201, 2002.

[2] M. Santini and R. Ricci, "Biventricular pacing in patients with heart failure and intraventricular conduction delay: state of the art and perspectives. The European view," Eur Heart J, vol. 23, no. 9, pp. 682686, 2002.

[3] D. Schäfer, J. Borgert, V. Rasche, and M. Grass, "Motion-compensated and gated cone beam filtered back-projection for 3-d rotational x-ray angiography." IEEE Transactions on Medical Imaging, vol. 25, no. 7, pp. 898-906, July 2006.
[4] D. Schäfer, U. Jandt, J. Carroll, and M. Grass, "Motion compensated reconstruction for rotational x-ray angiography using $4 \mathrm{~d}$ coronary centerline models," in 9th Int. Meeting on Fully 3D Image Reconstruction in Radiology and Nuclear Medicine, 2007, pp. 245-248, lindau, Germany.

[5] G. Lauritsch, J. Boese, L. Wigstrm, H. Kemeth, and R. Fahrig, "Towards cardiac c-arm computed tomography." IEEE Transactions on Medical Imaging, vol. 25, no. 7, pp. 922-934, July 2006.

[6] M. Li, H. Kudo, and H. Yang, "Improved 3d blood vessel reconstruction algorithm with gibbs smoothing prior from a limited number of projections," in 7th Int. Meeting on Fully 3D Image Reconstruction in Radiology and Nuclear Medicine, 2003, pp. Th AM2-3, saint Malo, France.

[7] E. Hansis, D. Schäfer, M. Grass, and O. Dössel, "An iterative method for the reconstruction of the coronary arteries from rotational x-ray angiography," in Medical Imaging 2007: Physics of Medical Imaging. Proceedings of the SPIE., J. Hsieh and M. J. Flynn, Eds., vol. 6510, no. 1, 2007, p. 651026.

[8] G. Shechter, F. Devernay, È. Coste-Manière, A. Quyyumi, and E. R. McVeigh, "Three-dimensional motion tracking of coronary arteries in biplane cineangiograms," IEEE Transactions on Medical Imaging, vol. 22, no. 4, pp. 493-503, 2003.

[9] C. Blondel, G. Malandain, R. Vaillant, and N. Ayache, "Reconstruction of coronary arteries from a single rotational x-ray projection sequence," IEEE Transactions on Medical Imaging, vol. 25, no. 5, pp. 653-663, may 2006, in press.

[10] U. Jandt, D. Schäfer, M. Grass, and V. Rasche, "Automatic generation of time resolved $4 \mathrm{~d}$ motion vector fields of coronary arteries," in 9th Int. Meeting on Fully 3D Image Reconstruction in Radiology and Nuclear Medicine, 2007, pp. 249-252, lindau, Germany.

[11] L. A. Feldkamp, L. C. Davis, and J. W. Kress, "Practical cone-beam algorithm," Journal of the Optical Society of America A, vol. 1, pp. 612-619, Jun. 1984.

[12] M. Zellerhoff, B. Scholz, E.-P. Ruehrnschopf, and T. Brunner, "Low contrast 3d reconstruction from c-arm data," in Medical Imaging 2005: Physics of Medical Imaging. Proceedings of the SPIE., M. J. Flynn, Ed., vol. 5745, Apr. 2005, pp. 646-655.

[13] M. Abramowitz and I. A. Stegun, Handbook of Mathematical Functions with Formulas, Graphs, and Mathematical Tables, 9th ed. New York: Dover, 1972.

[14] R. E. Klabunde, Cardiovascular Physiology Concepts. Lippincott Williams \& Wilkins, July 2004, iSBN 078175030X

[15] L. Husmann, S. Leschka, L. Desbiolles, T. Schepis, O. Gaemperli, B. Seifert, P. Cattin, T. Frauenfelder, T. G. Flohr, B. Marincek, P. A. Kaufmann, and H. Alkadhi, "Coronary Artery Motion and Cardiac Phases: Dependency on Heart Rate Implications for CT Image Reconstruction," Radiology, vol. 245, no. 2, pp. 567-576, 2007.

[16] M. Unser, "Splines: A perfect fit for signal and image processing," IEEE Signal Processing Magazine, vol. 16, no. 6, pp. 22-38, November 1999, iEEE Signal Processing Society's 2000 magazine award.

[17] G. Shechter, B. Shechter, J. R. Resar, and R. Beyar, "Prospective motion correction of x-ray images for coronary interventions." IEEE Transactions on Medical Imaging, vol. 24, no. 4, pp. 441-450, 2005.

[18] M. Prümmer, L. Wigstroem, J. Hornegger, J. Boese, G. Lauritsch, N. Strobel, and R. Fahrig, " Cardiac C-arm CT: Efficient Motion Correction for 4D-FBP ," in Nuclear Science Symposium, Medical Imaging , G. Smith, Ed., 2006, pp. 1-20.

[19] L. Ibanez, W. Schroeder, L. Ng, and J. Cates, The ITK Software Guide, 2nd ed., Kitware, Inc. ISBN 1-930934-15-7, http://www.itk.org/ItkSoftwareGuide.pdf, 2005.

[20] R. H. Byrd, P. Lu, J. Nocedal, and C. Zhu, "A limited memory algorithm for bound constrained optimization," SIAM J. Sci. Comput., vol. 16, no. 5, pp. 1190-1208, 1995. 


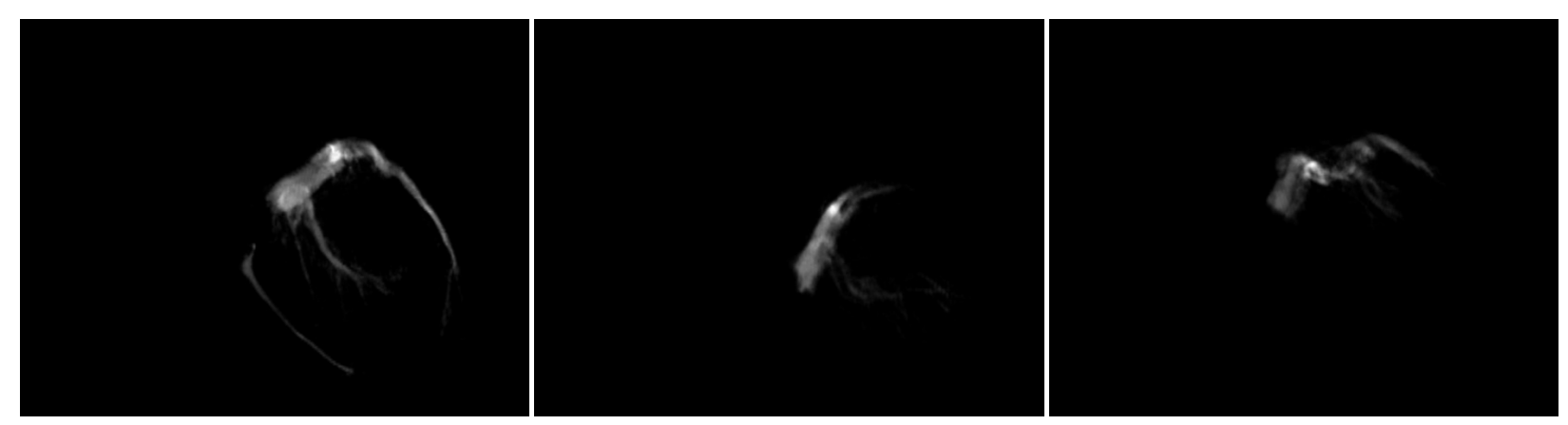

Standard reconstruction

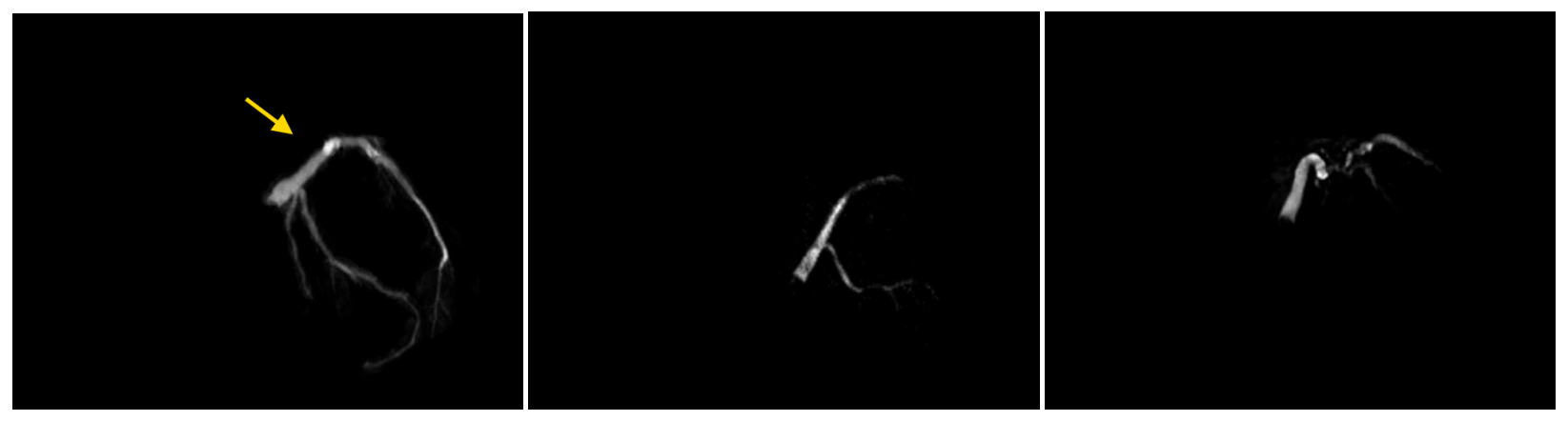

Gated streak reduced reconstruction
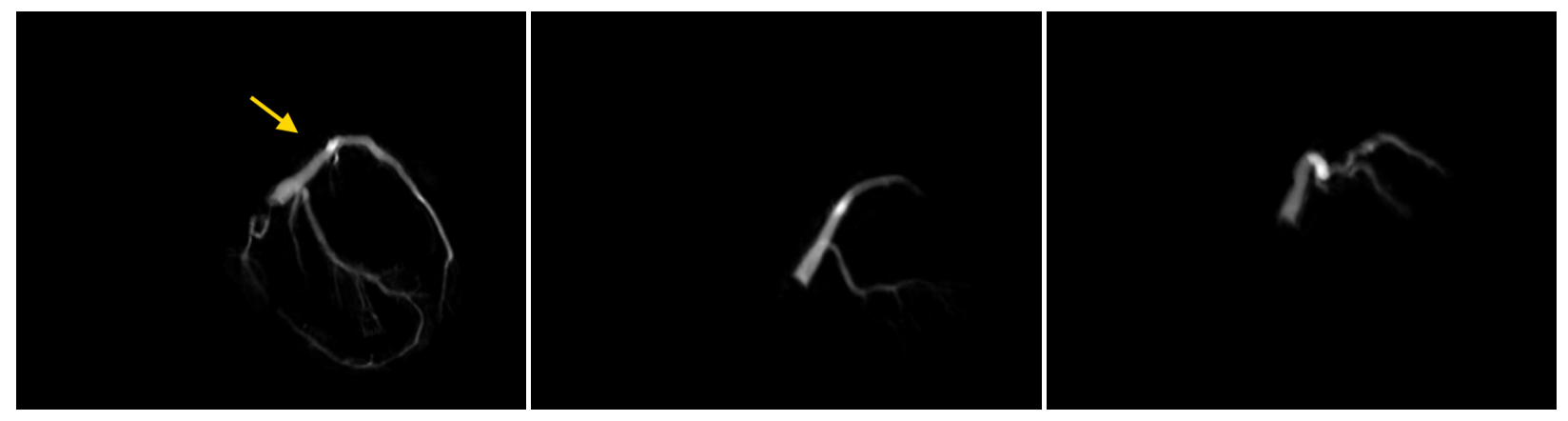

Motion compensated reconstruction

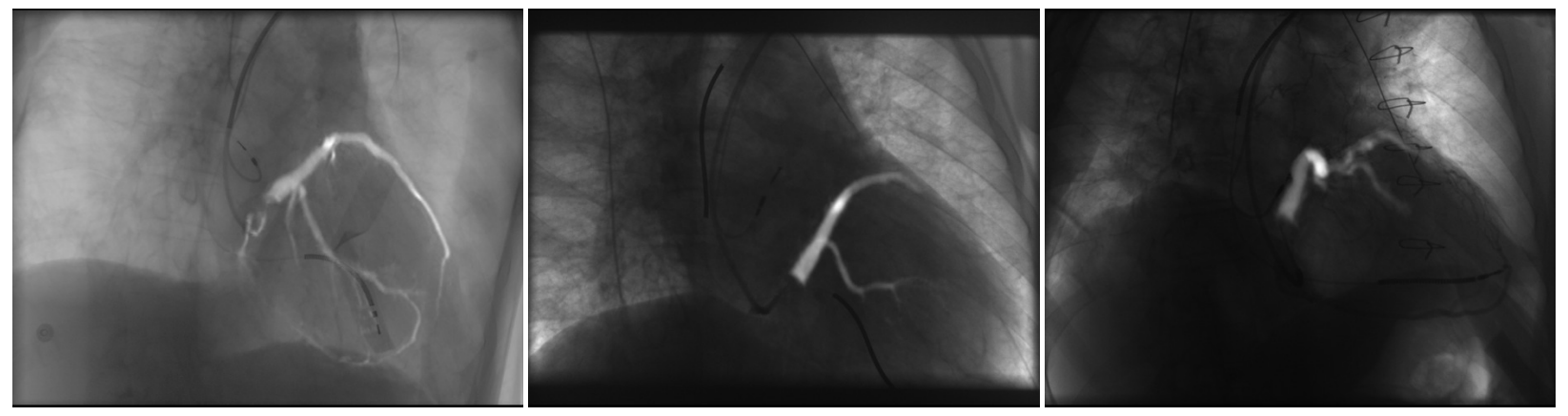

2-D/3-D overlay

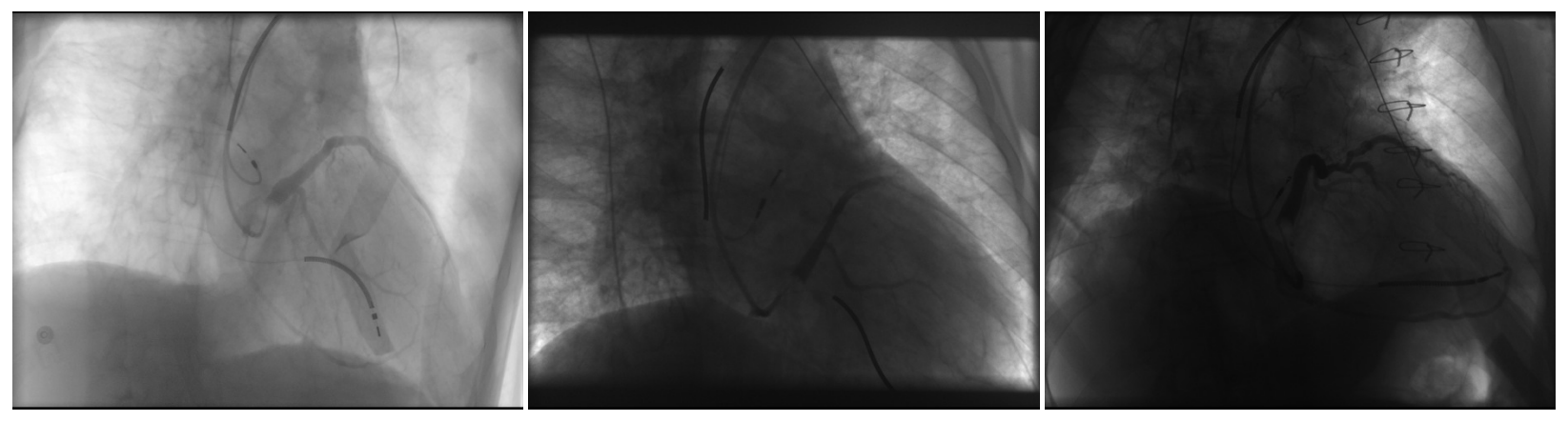

Projection image

Fig. 6: Forward projection of reconstruction results for three different clinical coronary sinus cases. Each of the cases is depicted column-wise from left to right. The arrow in the first case indicates the location of the extracted position displayed in figure 5. 